\title{
Osteonecrosis de los maxilares: fisiopatología, diagnóstico y tratamiento
}

\author{
Osteonecrosis of the jaws: pathophysiology, diagnosis and \\ treatment
}

María Catalina Cortés-Motta ${ }^{1 \otimes}$, Rafael Fernández-Grisales $^{1 \otimes} \underline{\text { CvLAC }}$

1. Endodoncista. Candidato a Maestría en Ciencias Odontológicas- Universidad CES, Medellín, Colombia.

Fecha correspondencia:
Recibido: junio de 2016.
Aceptado: noviembre de 2016.
Forma de citar:
Cortés-Motta M, Fernández-
Grisales R. Osteonecrosis de
los maxilares: fisiopatología,
diagnóstico y tratamiento. Rev. CES
Odont 2016; 29(2): 65-77.
Open access
C Derecho de autor
Licencia creative commons
Ética de publicaciones
Revisión por pares
Gestión por Open Journal System
ISSN 0120-971X
e-ISSN 2215-9185

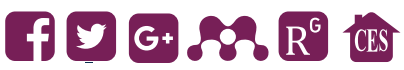

\section{Resumen}

El esqueleto humano es un conjunto organizado de piezas óseas que conforman y proporcionan soporte estructural al cuerpo. Diferentes funciones han sido descritas: soporte dinámico y mecánico, protección y mantenimiento de la homeostasis mineral. Para esto, el hueso debe mantener su metabolismo activo y ser capaz de adaptar su estructura a estímulos mecánicos reparando los daños estructurales a través del proceso de remodelación durante toda la vida. La osteoporosis, enfermedad de Paget, cáncer/infecciones en el hueso, van a alterar la fisiología del tejido dando lugar a la pérdida de su integridad. Los bifosfonatos (BFs) son agentes farmacológicos diseñados para el tratamiento de estas enfermedades, su principal mecanismo de acción es la inhibición de la resorción osteoclástica del hueso. La osteonecrosis de los maxilares (ONM) relacionada con la medicación consiste en la destrucción progresiva del tejido óseo, siendo uno de los efectos adversos de este tipo de tratamiento. Por esta razón, el objetivo de este artículo fue hacer una revisión acerca de los principales aspectos farmacológicos y clínicos de la ONM relacionada con los bifosfonatos en odontología. El diagnóstico clínico y el tratamiento adecuado son fundamentales para reducir el riesgo de osteonecrosis en pacientes bajo terapia antiresortiva o antes de iniciar su administración. Por esta razón, la prevención es aún más importante.

Palabras clave: Necrosis ósea, bifosfonatos, reabsorción ósea, remodelado óseo.

\section{Abstract}

The human skeleton is an organized set of bony pieces that make up and provide the structural support of the body. Different functions have been described: dynamic and mechanical support, protection and maintenance of mineral homeostasis. For this, the bone must maintain its active metabolism and be able to adapt its structure to mechanical stimuli repairing the structural damages through the process of remodeling throughout the life. Osteoporosis, Paget's disease, cancer and bone infections, will alter the physiology of the tissue resulting in the loss of its integrity. Bisphosphonates (BFs) are pharmacological agents designed for the treatment of these diseases, their main mechanism of action is the inhibition of bone 
osteoclastic resorption. Medication related osteonecrosis of the jaw $(\mathrm{ONJ})$ consists of the progressive destruction of the bone tissue, being one of the adverse effects of this type of treatment. For this reason, the objective of this article was to make a review about the main pharmacological and clinical aspects of ONJs related to bisphosphonates in dentistry. Clinical diagnosis and appropriate treatment are essential to reduce the risk of osteonecrosis in patients undergoing antiresorptive therapy or before starting administration. For this reason, prevention is even more important.

Keywords: Bone necrosis, bisphosphonates, bone resorption, bone remodeling.

\section{Introducción}

El esqueleto humano tiene en total 213 huesos y sus funciones específicas son: soporte y protección, sustento del movimiento, anclaje de los músculos y la homeostasis mineral. Es un tejido metabólicamente activo capaz de adaptar su estructura a los estímulos mecánicos y la reparación de daños estructurales a través del proceso de remodelación $(1,2)$. También se compone de hueso cortical $(80 \%)$ el cual es denso y sólido y rodea el espacio de la médula y hueso trabecular (20\%) que se compone de una red de placas trabeculares y varillas intercalados en el compartimento de la médula ósea. Tanto el hueso cortical y trabecular se componen de osteones $(1,2)$. El hueso maduro, se compone de colágeno tipo I que forma la matriz orgánica, uniéndose a los cristales de hidroxiapatita para realizar la mineralización. La matriz orgánica no colágena del hueso está compuesta por proteínas (osteocalcina, sialoproteinas, proteína morfogenética ósea- BMP, ILG1 e ILG2). Estas proteínas participan en el mantenimiento de la matriz ósea y la regeneración del hueso (ㅁ).

Los bifosfonatos han sido utilizados exitosamente para tratar enfermedades progresivas del esqueleto como: mieloma múltiple, metástasis ósea, osteoporosis y otras enfermedades metabólicas óseas (ㄴ). La primera asociación entre Bifosfonatos y ONMs fue descrita desde el 2003 (므). Las intervenciónes odontológicas quirúrgicas son asociadas como un factor de riesgo para ONMs. Por esto, el odontólogo debe identificar que pacientes son potenciales a desarrollar esta condición patológica, y establecer los protocolos de manejo adecuado antes, durante o posterior al uso de Bifosfonatos.

\section{Fisiología del hueso}

El hueso es un tejido conectivo altamente especializado y dinámico que se renueva constantemente. El modelado óseo es el proceso por el cual los huesos cambian su forma general en respuesta a influencias fisiológicas o fuerzas mecánicas, dando lugar a un cambio morfológico por la respuesta adaptativa al estrés, manteniendo la homeostasis mineral (1- $\underline{5})$. Las poblaciones celulares que participan en este proceso son: osteoblastos y osteoclastos, las cuales ejecutan la remodelación ósea y llevan a cabo secuencialmente la reabsorción de hueso viejo para evitar la acumulación de micro lesiones y generar la formación de nuevo hueso (1,2).

El ciclo de remodelación se compone de fases secuenciales: activación $\rightarrow$ reabsorción $\rightarrow$ formación. La activación implica el reclutamiento y activación de los osteoclastos (므). Los osteoclastos se derivan de células madre hematopoyéticas en la médula ósea y maduran como células mononucleares a través de la influencia de factores de transcripción c-Fos y PU.1 (Figura 1) (4). 


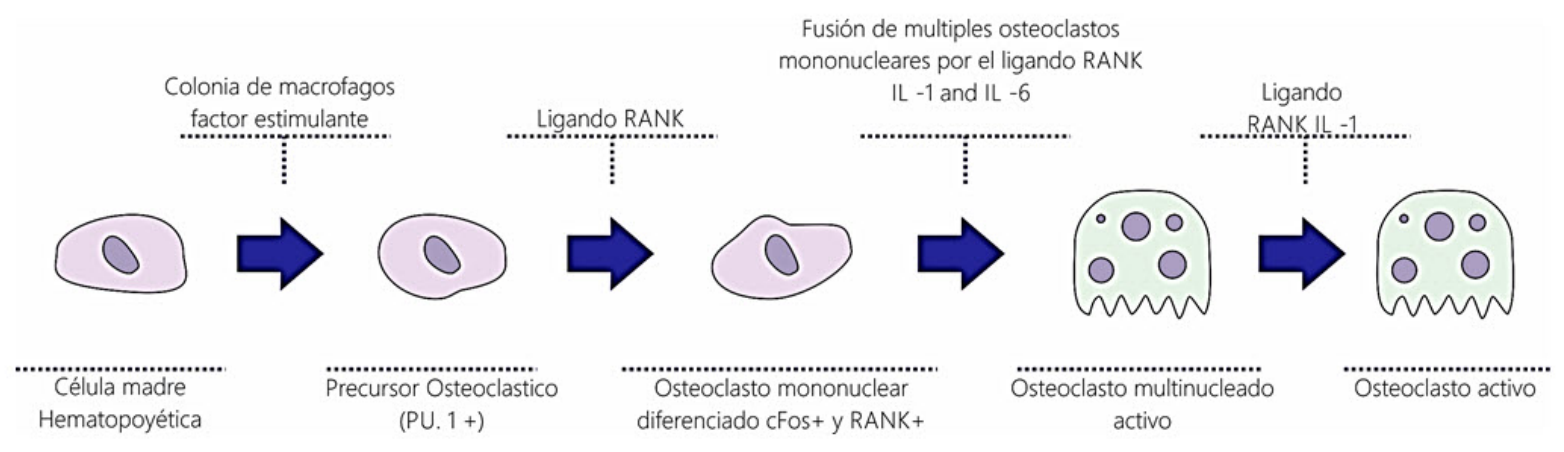

Figura 1. Proceso de maduración de un Odontoclasto. Imagen modificada de R.E. Marx (Ed.) 2006 (3)

La fusión de células de revestimiento de la superficie del hueso con las múltiples células mononucleares secretadas en la circulación por medio de la Interleucina-1; la Interleucina-6 y el receptor activador ligando Kappa-B (RANKL), forma los osteoclastos multinucleados $(\underline{1}, \underline{5})$. La reabsorción ósea mediada por osteoclastos tarda aproximadamente 2 a 4 semanas durante cada ciclo de remodelación. La formación de osteoclastos, la activación, y reabsorción están regulados por la relación de la activación a la respuesta de la hormona parotídea y del receptor ligando Kappa-B (RANKL); lo que resulta en la liberación de ácido clorhídrico a ( $\mathrm{pH}$ ácido $<1)$ que produce desmineralización de los cristales de hidroxiapatita y descomposición del componente orgánico de la matriz ( $99 \%$ colágeno) a través de la enzima colagenasa, que da lugar a las lagunas de Howship en la superficie del hueso trabecular y a los canales de Havers en el hueso cortical (1,4) (Figura 2). Después de la fase de reabsorción, se inicia la fase de renovación ósea. La liberación del ácido insoluble en la BMP y los factores de crecimiento similares a la insulina 1/2 (ILG1/ILG2), son liberados por los osteoclastos que promueven la diferenciación y proliferación de las células madre locales en osteoblastos. Estos osteoblastos recién diferenciados formarán una nueva matriz orgánica de colágeno, y secretarán sustancia osteoide. Posteriormente, estos osteoblastos maduros quedan sepultados dentro de la matriz mineralizada y se convertirán en osteocitos o células óseas de revestimiento con capacidad de difernciación por activación de la hormona paratiroidea o estímulos mecánicos (Figura 3). La unidad metabólica ósea (BMU) es la estructura anatómica temporal que comprende los osteoclastos y los osteoblastos que reemplazan los paquetes de hueso viejo con nuevo tejido óseo $(\underline{3}, \underline{4}, \underline{6})$. El mantenimiento de la función del tejido óseo requiere de una correcta integración de densidad y calidad ósea.

La formación de hueso tarda aproximadamente 4 a 6 meses y está siendo controlado por los osteoblastos que desarrollan la osteoprotegerina (OPG). La OPG, es una proteína inhibidora de la función de los osteoclastos, la cual esta en continúa competición con las proteínas estimulantes de los osteoclastos conocidos RANKL para evitar la unión con el receptor de membrana (RANK) del osteoclasto, evitando la resorción ósea y manteniendo el equilibrio óseo (므) (Figura 4). Una vez los osteoblastos y osteocitos cumplen su edad, la secreción de OPG disminuye permitiendo a los osteoclastos superar esta inhibición y ser capaces de reconocer hueso que es vulnerable a ser reabsorbido. Así, mantienen el esqueleto en una dinámica de renovación de hueso elástico joven capaz de responder a las tensiones que es sometido $(1,4)$ (Figura 5). 
Q

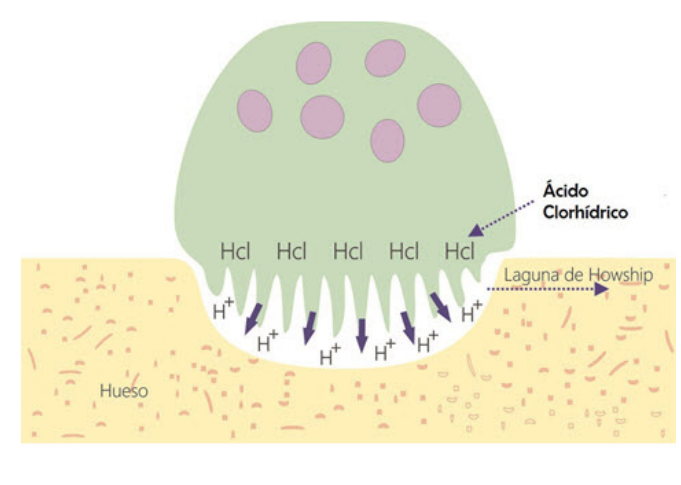

Figura 2. Reabsorción de hueso por los Osteoclastos unidos en la superficie del hueso, secretando ácido clorhídirco. El área reabsorbida del hueso es conocida como laguna de Howship.
Células madres mesenquimales

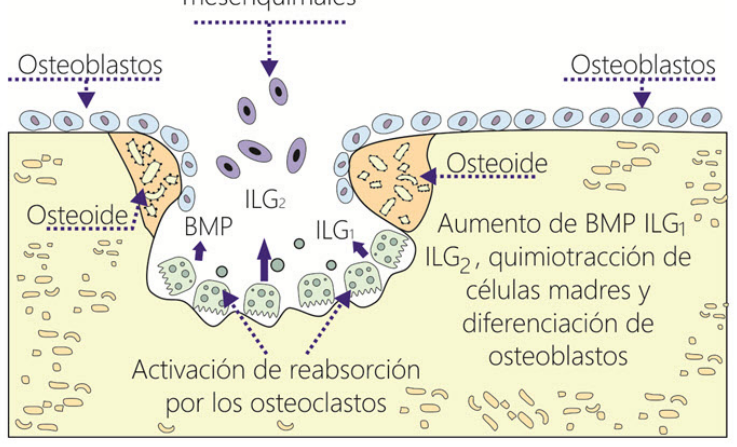

Figura 3. La reabsorción por odontoclastos promueve liberación de BMP, ILG-1, IGL-2 y otros factores causando la diferenciación de las células madre en odontoblastos.

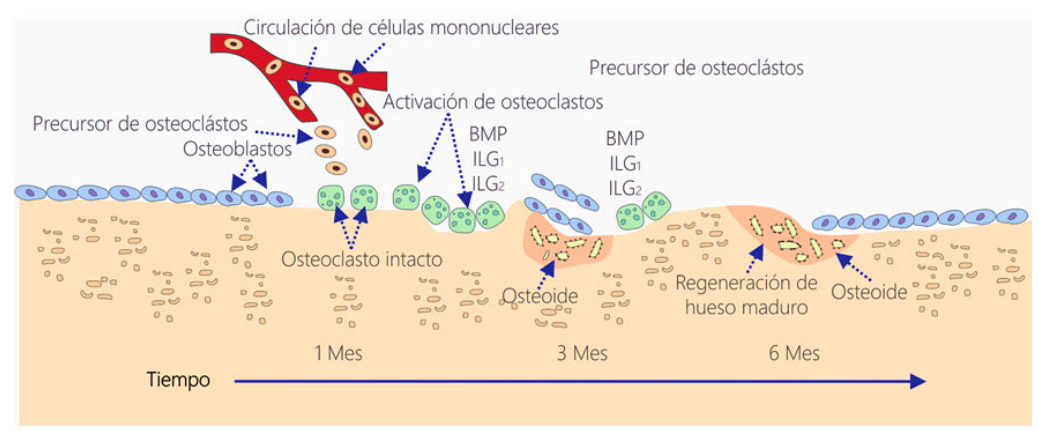

Figura 4. Ciclo de remodelación normal del hueso. Imagen adaptada de R.E. Marx (Ed.) 2006 (3).

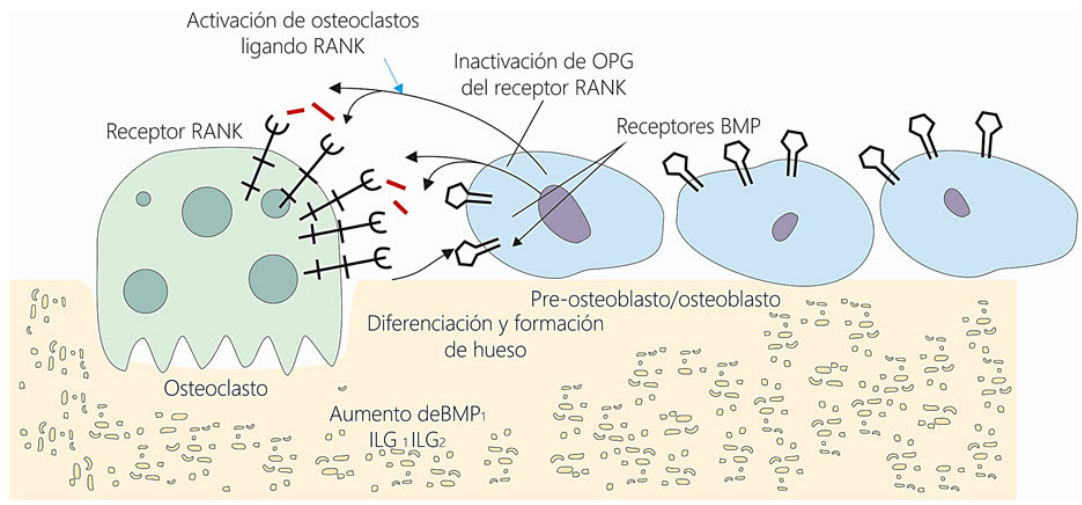

Figura 5. La competitiva inhibición y estimulación de los osteoclastos. La función autocrina de los osteoclastos y los efectos paracrinos de los Osteoblastos generan equilibrio en el proceso de renovación ósea. Imagen adaptada de R.E. Marx (Ed.) 2006 (3) 


\section{Bsifosfonatos}

\section{Estructura}

Los bifosfonatos (BFs) son análogos estructurales del pirofosfato (POP), con un carbono (PCP) reemplazando el oxígeno central (Figura 6). Son completamente resistentes a la hidrólisis, por lo tanto se acumulan en la matriz celular (ㄴ). Su molécula tiene dos cadenas laterales: R1 y R2, que varían su estructura dependiendo del producto. La R1 determina la unión a los cristales de hidroxiapatita del hueso (). La R2 determina las propiedades antirresortivas y puede dividirse en 2 clases: las que contienen o no el nitrógeno. La cadena que contienen nitrógeno aumenta su potencia y probablemente su toxicidad $(\underline{3}, \underline{8})$, siendo asociados a la inducción de ONMs. Los BFs son medicamentos usados para el tratamiento de trastornos óseos como: osteoporosis, enfermedad de Paget y en el control de síntomas y signos de malignidad ósea (Tabla 1) $(\underline{1}, \underline{3}, \underline{4}, \underline{8})$.

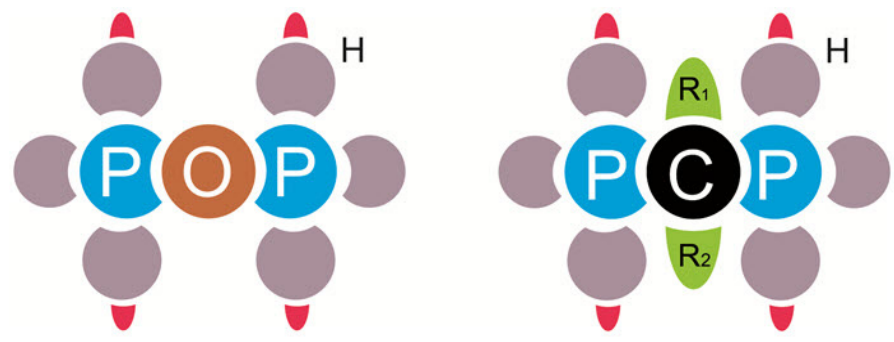

Q

Figura 6. Estructura tridimensional de los pirofosfatos y los bifosfonatos. El oxígeno es sustituido por el carbón haciéndola más resistente a la ruptura enzimática. Imagen adaptada de Goytia RN y col., 2009 (8).

Tabla 1. Tipos de Bifosfonatos aprobados para su uso por la FDA (3)

\begin{tabular}{|c|c|c|c|c|c|}
\hline Bifosfonato & Indicación Primaria & $\begin{array}{l}\text { Contenido de } \\
\text { Nitrogeno }\end{array}$ & Dosis & $\begin{array}{c}\text { Via } \\
\text { Administración }\end{array}$ & $\begin{array}{l}\text { Potencia } \\
\text { Relativa }\end{array}$ \\
\hline $\begin{array}{l}\text { Etidonato } \\
\text { (Didronel) }\end{array}$ & $\begin{array}{c}\text { Enfermedad de } \\
\text { Paget }\end{array}$ & No & $300-700 \mathrm{mg}$ diarios/6 meses & Oral & 1 \\
\hline $\begin{array}{l}\text { Tiludronato } \\
\text { (Skelid) }\end{array}$ & $\begin{array}{c}\text { Enfermedad de } \\
\text { Paget }\end{array}$ & No & $400 \mathrm{mg}$ diarios/3meses & Oral & 50 \\
\hline $\begin{array}{l}\text { Alendronato } \\
\text { (Fosamax) }\end{array}$ & Osteoporosis & Si & $10 \mathrm{mg} / \mathrm{dia} 70 \mathrm{mg} / \mathrm{semana}$ & Oral & 1000 \\
\hline Ibandronato (Boniva) & Osteoporosis & Si & $\begin{array}{c}2.5 \mathrm{mg} / \mathrm{dia} \\
150 \mathrm{mg} / \mathrm{mes}\end{array}$ & Oral & 1000 \\
\hline $\begin{array}{l}\text { Pamidronato } \\
\text { (Aredia) }\end{array}$ & Metastasis Ósea & Si & $90 \mathrm{mg} / 3$ semanas & Intravenosa & $1000-5000$ \\
\hline $\begin{array}{l}\text { Zolendronato } \\
\text { (Zometa) }\end{array}$ & Metastasis Ósea & Si & $4 \mathrm{mg} / 3$ semanas & Intravenosa & $1000+$ \\
\hline
\end{tabular}

\section{Bioactividad de los Bisfosfonatos}

La acción biológica de los BFs es inhibir la resorción ósea por medio del deterioro y la apoptosis de los osteoclastos. Por lo tanto, los beneficios clínicos resultan en la disminución neta del proceso normal de reabsorción ósea (2). Los BFs orales o intravenosos (IV) se adhieren fácilmente a los cristales minerales de la superficie ósea, por ende, las dosis repetidas se van acumulando en la matriz ósea. (Figura 7) $(3,4,6)$. La importancia de la estructura química radica en la presencia o no de nitrógeno en las cadenas laterales. Los BFs no nitrógenados, se metabolizan en 


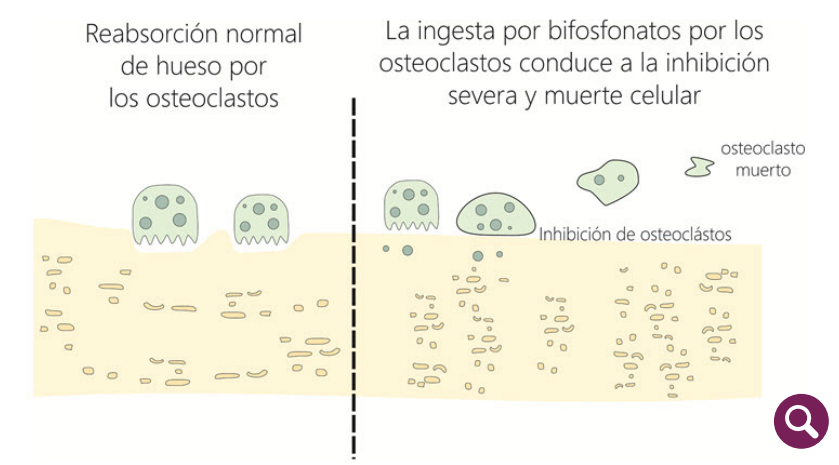

Figura 7. La ingesta de Bifosfonatos por los Osteoclastos en el proceso de reabsorción ósea causa muerte celular (apoptosis). Imagen adaptada de R.E. Marx (Ed.) 2006 (3). análogos no hidrozolubles de ATP, que luego se unen a minerales expuestos en el hueso y son absorbidos por los osteoclastos en la reabsorción del hueso. Los análogos de ATP se vuelven citotóxicos a medida que se acumulan estos subproductos, lo que lleva a la disminución de la función mitocondrial induciendo a la apoptosis $(\underline{3}, \underline{8}, \underline{9})$. De otro lado, los BFs no nitrógenados, aumentan su potencia inhibiendo la enzima farnesil pirofosfato sintasa (FPP) y geranil geranil pirofosfato (GGPP) dos enzimas clave en la vía mevalonato esenciales para la prenilación de proteínas (10). La Prenilación de proteínas permite la unión de pequeñas proteínas a la membrana celular y mediante la inhibición

de este proceso, los osteoclastos pierden la regulación celular y señalización generándose su apoptosis (9). El ácido Zoledrónico (Zoledronato), es un BFs de aplicación venosa, que se constituye como el más potente inhibidor de la reabsorción ósea (3).

\section{Farmacocinética de los Bifosfonatos}

Los BFs orales son absorbidos en el intestino delgado y solo 1-10\% están disponibles para el hueso, generando un efecto de goteo en la médula ósea y afectando menos a las células precursoras. Por el contrario, los BFs IV son de absorción inmediata al hueso 30-70\%, el resto es eliminado por los riñones. Existe una relación de absorción de los BFs IV y orales de 1/0.007, lo que significa que la acumulación y la absorción es 142.8 veces mayor para los BFs-IV que para los BFs-orales en el hueso. Su acumulación prolongada aumenta la toxicidad osteoclástica. (므, $4, \underline{6}$ ) (Figura 8).

Q

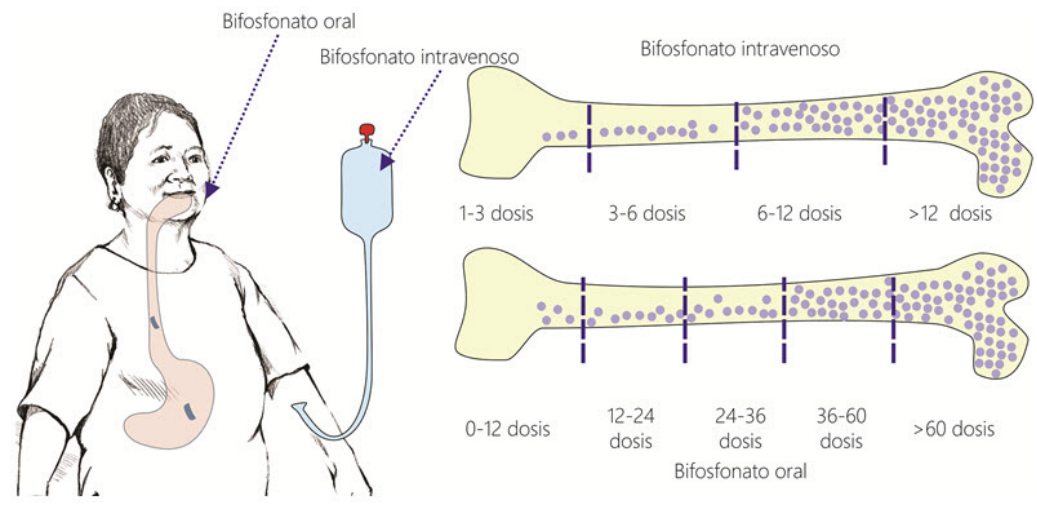

Figura 8. El acumulo de los BFs con nitrógeno oral/ intravenoso. Las dosis repetidas en un periodo continuo son más rápidas en BFs intravenosos que en los orales. Imagen adaptada de R.E. Marx (Ed.) 2006 (3).

\section{Osteonecrosis en maxilares relacionada a los Bisfosfonatos}

La Osteonecrosis es la exposición de hueso necrótico en los maxilares como un efecto secundario de la acción directa de los BFs (6). Se observa con mayor frecuencia en pacientes que han sido tratados con BFs IV y puede ocurrir espontáneamente (25\%), pero por lo general aumenta el riesgo con procedimientos médicos y odontológicos $(\underline{3}, \underline{6}, \underline{10}, \underline{11})$.

Existen diferentes definiciones para describir esta condición, siendo la más usada la de la Asociación Americana de Cirugía Maxilofacial (AAOMS-2007): "Osteone- 
crosis en maxilares relacionada a los Bifosfonatos" (ONMRM) (12). Sin embargo, la AAOMS-2014 sugiere cambiar la nomenclatura BRONJ por el término: "Osteonecrosis en los Maxilares Relacionada con Medicación" (ONMRM) debido al aumento continuo de casos de osteonecrosis que implican el maxilar y la mandíbula asociados con el uso de otros agentes antireabsortivos (13).

\section{Factores de riesgo}

ONMRM ocurre cuando la remodelación ósea es inhibida y no es capaz de mantener la homeostasis o respuesta en el sitio de la lesión (infección dentoalveolar, extracción dental, o trauma dental). El riesgo de ONMRM es significativamente mayor (95\%) para pacientes que reciben BFs IV, por el contrario, es menor para pacientes que reciben BFs orales posiblemente por su baja absorción (menor al 1\%) siendo el principal factor de riesgo $(\underline{3}, 11,14)$ (Tabla 2). Otro factor de riesgo potencial entre otros (Tabla 3), es la duración y dosificación del tratamiento, ya que cada año el riesgo de desarrollar ONMRM aumenta el 57\% (14). La acumulación de bifosfonatos altera e inhibe la remodelación ósea, hay disminución del suplemento sanguíneo, lo que conduce a la isquemia, necrosis e infección causando necrosis ósea $(\underline{4}, \underline{6}, \underline{15}, 16)$.

Tabla 2. Diferencias entre osteonecrosis por bifosfonatos orales e intravenosos.

\begin{tabular}{lcc}
\hline & Bisfosfonatos intravenosos & Bisfosfonatos orales \\
\hline Incidencia & $0,8-12 \%$ & $0,01-0,04 \%$ \\
Tiempo de Administración & Corto: $9,3-14,1$ meses & Largo: 3,3-10,2 años \\
Localización & Mandíbula/maxilar sector posterior & Mandíbula Sector posterior \\
Tamaño de exposición: & mayor tamaño & Menor Tamaño \\
Antecedente quirúrgico & $70 \%$ & $50 \%$ \\
Evolución & Impredecible & Favorable \\
\hline
\end{tabular}

Tabla 3. Factores de riesgo de ONMRM

\section{Factores de riesgo locales}

a. Cirugías: Exodoncias, Implantes, Cirugía periapical y periodontal. Los bifosfonatos inhiben la migración y proliferación de células epiteliales, disminuyendo proceso de cicatrización aumentando el riesgo de infección bacteriana. La activación del remodelado óseo es esencial para la cicatrización $(7,11,18)$.

b. Infección local: Las enfermedades inflamatorias inducen remodelado óseo contínuo. El $84 \%$ de los pacientes con osteonecrosis inducida por bifosfonatos intravenosos, presentaban: periodontitis activa, $29 \%$ caries dental, 13\% absceso dental, 11\% tratamiento endodóntico defectuoso. $(4,11,19)$

c. Historia de trauma: La oclusión causa tensión y compresión de las fibras del ligamento periodontal; la lámina dura establece un hueso más denso que es capaz de soportar la tensión, sin embargo, los bifosfonatos previenen la reabsorción lo que resulta en una hipermineralización de la lámina dura con hueso no remodelado $(4,13,14,15)$.

d. Sistémicos: Pacientes con cáncer tienen mayor riesgo debido a que reciben tratamientos que afectan su sistema inmune, además están frecuentemente administrados con bifosfonatos intravenosos para el tratamiento de complicaciones óseas incluyendo metástasis óseas, dolor óseo e hipercalcemia (13). La diabetes y VIH disminuyen el sistema inmune, aumentando la incidencia de ONMRM (21)

e. Otros factores de riesgo: La mala higiene oral, el tabaquismo y alcohol no solo aumentan la incidencia, sino que también exacerba las condiciones. 


\section{Incidencia}

Los maxilares por sus características clínicas y anatómicas están asociados a ser las zonas con mayor incidencia de osteonecrosis. El hueso alveolar depende más de la remodelación y renovación osteoclástica que cualquier otro hueso del esqueleto humano. El efecto citotóxico de los bisfosfonatos sobre los osteoclastos y las células del ligamento periodontal suprimen el recambio óseo. Los bisfosfonatos tienden a concentrarse mayor en mandíbula debido a su alta vascularización, causando un efecto antiangiogenico. Además, existe una alta diversidad de la microbiota oral siendo el ambiente ideal para una rápida proliferación bacteriana $(\underline{4}, 15$ ). La primera exposición ósea después de la administración de bifosfonatos es de aproximadamente 14,3 meses (pamidronato), 12,1 meses (pamindronato y zoledronato), 9,4

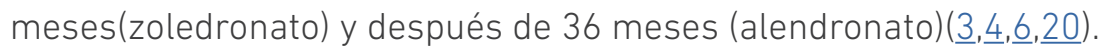

\section{Signos y síntomas}

La osteonecrosis se manifiesta como una exposición de hueso necrótico. El 68,1\% de los casos son exclusivamente en mandíbula, 27,7\% en maxilar, 4,2 \% simultaneo en mandíbula y maxilar( $\underline{3}, \underline{4}, \underline{6})$. La zona retro molar mandibular con un $65,5 \%(\underline{6}, 14)$. La Asociación Americana de Cirugía Maxilofacial dio estados de clasificación para el MRONM (14) (Tabla 4).

Tabla 4. Estados de ONMRM según la AAMOS-2007 (13)

\begin{tabular}{cl}
\hline Estados de Alarma & Estados de MRONM \\
\hline Estado $\mathbf{0}$ & $\begin{array}{l}\text { No hay apariencia de exposición/necrosis del hueso- pequeñas lesiones osteoliticas en la radiografía } \\
\text { Estado } \mathbf{1}\end{array}$ \\
$\begin{array}{l}\text { Exposición hueso de un cuadrante o menos, sin osteolisis más allá del alveolo-asintomático, no hay presencia } \\
\text { de infección }\end{array}$ \\
Estado 2 & $\begin{array}{l}\text { Exposición de hueso en dos o más cuadrantes, sin evidencia de osteolisis más allá del alveolo- presencia de } \\
\text { infección(dolor-inflamación) }\end{array}$ \\
\hline
\end{tabular}

\section{Examen radiográfico}

La radiografía panorámica generalmente muestra áreas de radiolucidez (osteolísis) y radiopacidad (osteoclerosis) mal definidas. También, secuestros óseos, esclerosis o pérdida de la lámina dura y/o ensanchamiento del espacio del ligamento periodontal $(\underline{22}, \underline{23})$. Otro tipo de radiografía como la TC (Tomografía Computarizada), pueden revelar con mayor claridad el estado de destrucción del hueso cortical buco-lingual, y cuando la lesión es indetectable clínicamente puede ser útil para el diagnóstico de estado temprano de $\operatorname{ONMRM}(\underline{3}, \underline{6})$.

\section{Diagnóstico diferencial}

Las enfermedades asociadas con exposición de hueso sin historia de tratamiento con bisfosfonatos, son diagnósticos diferenciales para ONMRM (alveolitis o alveolo seco, sinusitis, gingivitis, lesiones periapicales, desorden temporo-mandibular, tumores y metástasis de tumor primario a la mandíbula). Es necesario realizar un examen histopatológico de las lesiones de ONMRM para poder descartar diagnósticos diferenciales (7). Hay que tener en cuenta que al examen radiográfico se observan lesiones muy similares a la osteonecrosis, entre ellas, la osteomielitis y la osteoradionecrosis.

\section{Marcadores metabólicos para ONMRM}

El diagnóstico de ONMRM puede hacerse con la historia de tratamiento con bifosfonatos, hallazgos clínicos de las lesiones orales macroscópicas, e imágenes radio- 
gráficas. Sin embargo, Marx. Y col. encontró relación entre los niveles sanguíneos de C-Telopeptide (CTX) y la administración de bifosfonatos (4). El CTx que es un marcador bioquímico de la reabsorción ósea que mide una secuencia de ocho aminoácidos de colágeno liberados por los osteoclastos. La disminución de los niveles de colágeno $1 \mathrm{C}$ refleja la citoxicidad en los osteoclastos por el efecto de los bifosfonatos $(13,14)$. Los valores normales de los niveles de CTx en adultos oscilan entre 350 y $500 \mathrm{pg} / \mathrm{ml}$, y en pacientes medicados con bifosfonatos oscila entre 20 a $55 \mathrm{pg} / \mathrm{ml}$, dependiendo del medicamento específico, la dosis, y el número de dosis tomadas. La utilidad clínica es muy limitante, en pacientes con cáncer no son fiables por la invasión a tejidos y su constante descomposición de colágeno, al igual que en pacientes que toman corticoesteroides en enfermedades inmunes ya que estos medicamentos reducen el número de células madres de la médula ósea, alterando los resultados. La elevación de niveles en sangre de CTx mayor que $150 \mathrm{pg} / \mathrm{ml}$ seguido de la discontinuidad de los bifosfonatos, es asociado con la recuperación de la reabsorción, la cirugía dental bajo estas condiciones no causa ONRBF, ya que es suficiente para la curación ósea (15). Sin embargo, niveles de CTx $<100-125 \mathrm{pg} / \mathrm{ml}$ presentan un riesgo de moderado a alto para osteonecrosis en mandíbula (13).

\section{Cáncer y Osteoporosis - relación con Osteonecrosis de los maxialares}

Los bifosfonatos IV son ampliamente usados para tratamiento del mieloma múltiple y metástasis ósea en cáncer de mama y próstata. Su incidencia 0,8-1,2\% de ONRBF en pacientes medicados con bifosfonatos IV, puede aumentar al $21 \%$ cuando el bifosfonato es administrado por más de 3 años. La discontinuidad de los bifosfonatos en pacientes con cáncer es difícil, el tratamiento de la enfermedad maligna debe ser siempre la prioridad incluso si ONMRM ocurre (14).

La Osteoporosis es una enfermedad sistémica metabólica caracterizada por la pérdida de masa y el deterioro de la micro-arquitectura ósea, llevando a la fragilidad del hueso y susceptibilidad de fractura (17). Los bifosfonatos son los medicamentos de elección para estabilizar la pérdida ósea en mujeres menopaúsicas. El objetivo del tratamiento para la osteoporosis es inhibir el proceso de resorción del hueso mediada por los osteoclastos, aumentar la mineralización y preservar la densidad ósea. Los medicamentos actuales para la osteoporosis son: suplementos de calcio, vitamina D, bifosfonatos, estrógeno selectivo, calcitonina, hormona paratiroide (Teriparatide) y denosumab (23). A pesar del mecanismo de acción de los bifosfonatos, la incidencia de que ocurra ONRBF se estima que sea de 0,01-0,04\% en pacientes tratados con alendronato una vez a la semana y aumenta de 0,09-0,34\% después de la extracción dental, lo que resulta en una incidencia de ONRBF muy baja en pacientes con osteoporosis $(\underline{3}, \underline{4}, \underline{22}, \underline{24})$.

\section{Cuidados preventivos antes/durante la administración de Bisfosfonatos}

Es recomendable tener una guía para el manejo en la consulta odontológica en pacientes que van o están siendo medicados con bifosfonatos orales/ intravenosos. Los procedimientos preventivos son de gran importancia para reducir el riesgo de desarrollar ONRBF. Una vez el paciente es informado del tratamiento con bifosfonatos, esté debe ser remitido a valoración y tratamiento odontológico (25). Una buena comunicación entre médico, odontólogo y paciente es importante, pero también la educación al paciente sobre el posible riesgo de desarrollar ONMRM $(\underline{11}, \underline{21}, \underline{26})$ (Tabla 5). 
Tabla 5. Cuidados preventivos antes y durante la administración de BFs $(14,21,22,24)$

Antes de administración de BFs

Durante la administración de BFs

1. Examen radiográfico (panorámica y periapical completo)

2. Eliminar focos infecciosos para prevenir procedimientos en un futuro cercano:

- Exodoncias

- Cirugías periodontales

- Endodoncia

- Control de caries

- Prótesis (eliminar áreas donde está produciendo mayor presión o fricción)

3. Proceso Cicatrización de 1 mes

4. Instrucción y motivación en higiene oral.

5. Para pacientes con cáncer, la terapia antibiótica profiláctica es necesaria.

6. Controles periódicos.

7. Los bifosfonatos deben iniciar una vez terminado el tratamiento odontológico completo
1. Examen radiográfico (panorámica y periapical completo)

2. Instrucción y motivación en higiene oral.

3. Control de la caries -restauraciones

4. Tratamiento endodóntico no quirúrgico si es necesario.

5. Si los dientes no son restaurables por caries el tratamiento endodóntico no quirúrgico y amputación de la corona es la mejor opción

6. Si el diente presenta movilidad grado 1 y 2 realizar ferulización.

7. Si la movilidad es mayor a 3 existe una alta posibilidad de que la oteonecrosis está presente y el absceso y granulación está tapando el hueso expuesto. En este caso la exodoncia y terapia antibiótica es el único recurso.

\section{Tratamiento para osteonecrosis en maxilares relacionada con bisfosfonato}

Los objetivos terapéuticos para el manejo ONMRM están encaminados a mejorar la calidad de vida de los pacientes $(\underline{3}, \underline{20}, \underline{21})$. La AAMOS y la JADA establecieron estrategias de tratamiento para cada estadio del MRONM $(\underline{4}, 12,18)$ (Tabla 6).

Tabla 6. Tratamiento para pacientes con ONMRM según su estadio. $(4,11)$

\begin{tabular}{|c|c|}
\hline Estadio MRONM & Tratamiento \\
\hline Estadio 0 & $\begin{array}{l}\text { No hay tratamiento indicado; educación e instrucción en higiene oral. Eliminar cualquier } \\
\text { causa inflamatoria dental. }\end{array}$ \\
\hline Estadio 1 & $\begin{array}{l}\text { Enjuague bucal (Clorhexidina al 0,12\%/3 veces al día). } \\
\text { Educación, motivación e instrucción en higiene oral. Seguimiento clínico. }\end{array}$ \\
\hline & $\begin{array}{l}\text { Enjuague bucal ( } 0,12 \% \text { de Clorhexidina/3 veces al día) } \\
\text { Tratamiento sistémico con antibióticos. } \\
\text { 1. Penicilina } V-K 500 \mathrm{mg} / 4 \text { veces al día continuo o hasta que el dolor e infección es } \\
\text { controlado } \\
\text { Alérgicos a penicilina o no responde a esta: }\end{array}$ \\
\hline Estadio2 & $\begin{array}{l}\text { 2. Doxiciclina una vez al día } 100 \mathrm{mg} \text { continuo o hasta que el dolor e infección es } \\
\text { controlado } \\
\text { Levofloxacin } 500 \mathrm{mg} \text { una vez al día durante la fase sintomática. } \\
\text { Azitromicina } 500 \mathrm{mg} \text { una vez al día durante la fase sintomática. } \\
\text { Educación e instrucción en higiene oral. } \\
\text { No se recomienda realizar desbridamiento por el riesgo de exposición hueso. }\end{array}$ \\
\hline Estadio 3 & $\begin{array}{l}\text { Igual manejo del estadio 2. Es necesario recesión ósea (Alveolectomía) o en casos muy } \\
\text { extremos mandibulectomia }\end{array}$ \\
\hline
\end{tabular}

\section{Conclusiones}

En el futuro es fundamental mejorar los conocimientos actuales sobre MRONM y desarrollar mejores estrategias para su prevención y tratamiento. Las recomendaciones clínicas de este informe, se basan en una evaluación crítica de la evidencia científica que deben integrarse con el juicio y el conocimiento del profesional, las necesidades y preferencias del paciente no representan un estándar de atención. 


\section{Referencias bibliográficas}

1. Clarke B. Normal Bone Anatomy and Physiology. Clin J Am Soc Nephrol. 2008 Nov 1;3(Supplement 3):S131-9. https://www.ncbi.nlm.nih.gov/pubmed/18988698

2. Robling $A G$, Castillo $A B$, Turner $\mathrm{CH}$. Biomechanical and molecular regulation of bone remodeling. Annu Rev Biomed Eng. 2006;8:455-498. https://www.ncbi. nlm.nih.gov/pubmed/16834564

3. R.E. Marx (Ed.) Oral and Intravenous Bisphosphonate-Induced Osteonecrosis of the Jaws: History, Etiology, Prevention, and Treatment. Quintessence Publishing Co Inc, Second edition Hanover Park, IL; 2006. http://www.quintpub.com/display detail.php3?psku=B5105\#.WloXp I Oko

4. Marx RE. A Decade of Bisphosphonate Bone Complications: What It Has Taught Us About Bone Physiology. Int J Oral Maxillofac Implants. 2014;29(2):e247-58. https://www.ncbi.nlm.nih.gov/pubmed/24683588

5. Parfitt AM. Quantum concept of bone remodeling and turnover: implications for the pathogenesis of osteoporosis. 1979;28:1-5. https://www.ncbi.nlm.nih.gov/ pubmed/115547

6. Marx RE, Sawatari Y, Fortin M, Broumand V. Bisphosphonate-Induced Exposed Bone (Osteonecrosis/Osteopetrosis) of the Jaws: Risk Factors, Recognition, Prevention, and Treatment. J Oral Maxillofac Surg. 2005; 63(11):1567-75. https:// www.ncbi.nlm.nih.gov/pubmed/16243172

7. Durdica Grbesa TB. Pathophysiology of Osteonecrosis of the Jaw in Patients Treated with Bisphosphonate. Review. 2013;37(2):645-51. https://www.ncbi. nlm.nih.gov/pubmed/23941019

8. Goytia RN, Salama A, Khanuja HS. Bisphosphonates and Osteonecrosis: Potential Treatment or Serious Complication? Orthop Clin North Am. 2009 Apr;40(2):22334. https://www.ncbi.nlm.nih.gov/pubmed/19358907

9. Rasmusson L, Abtahi J. Bisphosphonate Associated Osteonecrosis of the Jaw: An Update on Pathophysiology, Risk Factors, and Treatment. Int J Dent. 2014;1-9 https://www.hindawi.com/journals/ijd/2014/471035/

10. Aksel H, Serper A. Recent considerations in regenerative endodontic treatment approaches. J Dent Sci. 2014; 9(3):207-13. http://www.sciencedirect.com/ science/article/pii/S1991790214000348

11. Helen Ristic JWH, Robert A. Adler, Beatrice Edwuards, Peter L. Jacobsen, Sreenivas Koka, Cesar A. Migliorati. Managing the care of patients receiving antiresorptive therapy for prevention and treatment of osteoporosis: Executive summary of recommendations from the American Dental Association Council on Scientific Affairs. JADA. 2011:142:1243-51. https://www.ncbi.nlm.nih.gov/ pubmed/22041409

12. Silverman SL, Landesberg R. Osteonecrosis of the Jaw and the Role of Bisphosphonates: A Critical Review. Am J Med. 2009 Feb;122(2):S33-45. https://www. ncbi.nlm.nih.gov/pubmed/19187811 
13. Ruggiero SL, Dodson TB, Fantasia J, Goodday R, Aghaloo T, Bhoomi M, O'Ryan F. American Association of Oral and Maxillofacial Surgeons position paper on medication-related osteonecrosis of the jaw-2014 update. J Oral Maxillo Fac Surg 2014;72:1938-56.

14. Yoneda T, Hagino H, Sugimoto T, Ohta H, Takahashi S, Soen S, et al. Bisphosphonate-related osteonecrosis of the jaw: position paper from the Allied Task Force Committee of Japanese Society for Bone and Mineral Research, Japan Osteoporosis Society, Japanese Society of Periodontology, Japanese Society for Oral and Maxillofacial Radiology, and Japanese Society of Oral and Maxillofacial Surgeons. J Bone Miner Metab. 2010;28(4):365-83. https://www.ncbi.nlm.nih. gov/pubmed/20333419

15. Lodi G, Sardella A, Salis A, Demarosi F, Tarozzi M, Carrassi A. Tooth Extraction in Patients Taking Intravenous Bisphosphonates: A Preventive Protocol and Case Series. J Oral Maxillofac Surg. 2010;68(1):107-10. https://www.ncbi.nlm.nih. gov/pubmed/20006163

16. Subramanian G, Cohen HV, Quek SYP. A model for the pathogenesis of bisphosphonate-associated osteonecrosis of the jaw and teriparatide's potential role in its resolution. Oral Surg Oral Med Oral Pathol Oral Radiol Endodontology. 2011;112(6):744-53. https://www.ncbi.nlm.nih.gov/pubmed/21821444

17. Linares JG. Efectividad del legrado óseo quirúrgico como tratamiento de la osteoquimionecrosis mandibular. [cited 2014 Nov 9]; Available from: https://ddd. uab.cat/pub/tesis/2013/hdl_10803_125968/jgl1de1.pdf

18. Moinzadeh A-T, Shemesh H, Neirynck NAM, Aubert C, Wesselink PR. Bisphosphonates and their clinical implications in endodontic therapy. Int Endod J. 2013;46(5):391-8. https://www.ncbi.nlm.nih.gov/pubmed/23137312

19. Martins CA, Leyhausen G, Volk J, Geurtsen W. Effects of Alendronate on Osteoclast Formation and Activity In Vitro. J Endod [Internet]. 2014 Oct [cited 2014 Nov 9]; Available from: http://linkinghub.elsevier.com/retrieve/pii/S009923991400644X

20. Fleisher KE, Welch G, Kottal S, Craig RG, Saxena D, Glickman RS. Predicting risk for bisphosphonate-related osteonecrosis of the jaws: CTX versus radiographic markers. Oral Surg Oral Med Oral Pathol Oral Radiol Endodontology. 2010;110(4):509-16.

21. AAE. American Association of Endodontists. Endodontic implications of bisphosphonate-associated osteonecrosis of the jaws. 2008 Jul 10; Available from: http://www.tupeloendo.com/pdfs/Bisphosphonate- Associated-Osteonecrosis. pdf. Accessed

22. Fleisher KE, Welch G, Kottal S, Craig RG, Saxena D, Glickman RS. Predicting risk for bisphosphonate-related osteonecrosis of the jaws: CTX versus radiographic markers. Oral Surg Oral Med Oral Pathol Oral Radiol Endodontology. 2010;110(4):509-16. https://www.ncbi.nlm.nih.gov/pubmed/20674404

23. Otomo-Corgel J. Osteoporosis and osteopenia: implications for periodontal and implant therapy. Periodontol 2000.2012;59:111-139. https://www.ncbi.nlm.nih.gov/pubmed/22507063 
24. Bittner T, Lorbeer N, Reuther T, Böhm H, Kübler AC, Müller-Richter UDA. Hemimandibulectomy after bisphosphonate treatment for complex regional pain syndrome: A case report and review on the prevention and treatment of bisphosphonate-related osteonecrosis of the jaw. Oral Surg Oral Med Oral Pathol Oral Radiol. 2012;113(1):41-7. https://www.ncbi.nlm.nih.gov/pubmed/22677690

25. Borromeo G, Tsao C, Darby I, Ebeling P. A review of the clinical implications of bisphosphonates in dentistry: Dental implications of bisphosphonates. Aust Dent J. 2011;56(1):2-9. https://www.ncbi.nlm.nih.gov/pubmed/21332734

26. Fleisher KE, Welch G, Kottal S, Craig RG, Saxena D, Glickman RS. Predicting risk for bisphosphonate-related osteonecrosis of the jaws: CTX versus radiographic markers. Oral Surg Oral Med Oral Pathol Oral Radiol Endodontology. 2010;110(4):509-16. https://www.ncbi.nlm.nih.gov/pubmed/20674404 